\title{
Drug-Drug Interactions in Patients with COVID-19 in Nursing Homes
}

\author{
Florian Correard ${ }^{1} \cdot$ Anne-Laure Couderc $^{2} \cdot$ Robin Arcani $^{2} \cdot$ Joris Weiland $^{2} \cdot$ Anais Courcier $^{2} \cdot$ Charlotte Berard $^{1}$. \\ Charlène Miola ${ }^{2} \cdot J_{\text {Julie Berbis }}^{3} \cdot$ Patrick Villani $^{2} \cdot$ Aurélie Daumas $^{2} \mathbb{D}$
}

Accepted: 11 March 2021 / Published online: 31 March 2021

(C) The Author(s), under exclusive licence to Springer Nature Switzerland AG 2021

\section{Dear Editor}

We read with great interest the recent article entitled "Drug-Drug Interactions and Prescription Appropriateness in Patients with COVID-19: A Retrospective Analysis from a Reference Hospital in Northern Italy" written by Cattaneo et al. [1] in which the authors highlighted that the addition of specific treatments for coronavirus disease 2019 (COVID-19) in patients with polypharmacy increases the risk of drug-drug interactions (DDIs). In this article [1], the authors provided data supporting the risk of severe DDIs in 399 hospitalized patients with COVID-19 (50\% aged $>65$ years) treated with at least two drugs, by the use of lopinavir/ritonavir and/or hydroxychloroquine and/or antibiotics. During hospitalization, one potential DDI was noted in $85 \%$ of patients and was classified as potentially severe in $80 \%$ of cases. It is worth noting that the majority of potentially severe DDIs carried an increased risk of QT prolongation, which accounted for almost $90 \%$ of the predicted DDIs. About one-quarter of contraindicated DDIs observed were hydroxychloroquine-induced DDIs and $>50 \%$ could be attributed to lopinavir/ritonavir. An increase in antibiotic consumption was also noted with the use in particular of azithromycin, which also increases the risk of QT prolongation.

Hodge et al. [2] also confirmed that COVID-19 therapies led to a significant risk for DDIs. In their review until 30

Aurélie Daumas

aurelie.daumas@ap-hm.fr

1 Pharmaceutical Expertise and Clinical Research Unit, Pharmacy Department, Timone Hospital, AP-HM, AixMarseille University, Marseille, France

2 Internal Medicine, Geriatry and Therapeutic Department, Timone and Sainte Marguerite Hospital, AP-HM, Aix-Marseille University, CHU Timone, 264 Rue Saint Pierre, 13384 Marseille, France

3 School of Medicine-La Timone Medical Campus, EA 3279: CEReSS Health Service Research and Quality of Life Center, Aix-Marseille University, Marseille, France
June, 2020, drug interactions involving lopinavir/ritonavir were the most frequent, followed by chloroquine or hydroxychloroquine. To increase recognition of harmful drug interactions and promote safe prescribing, the authors proposed a DDI online resource on www.covid19-druginteractions.org.

In nursing homes, to the best of our knowledge, the risk of DDIs has not been assessed while residents are prescribed more medications than older patients in any other medical setting because of the number and severity of chronic medical comorbidities [3, 4]. Indeed, the highest rate of polypharmacy occurs in nursing home residents [3, 4]; thus each new medication should be prescribed cautiously.

We retrospectively analyzed 480 residents (mean age 88 years, range 65-105 years) with COVID-19 managed in 15 nursing homes in the south of France between 1 March and 20 May, 2020. All residents had at least one comorbidity, primarily neurocognitive disorders affecting $84.6 \%$ of residents, hypertension $(53.5 \%)$, and depression $(45.0 \%)$. Fifty-one residents $(10.6 \%)$ had a chronic pulmonary disease and $17.9 \%$ of residents had diabetes mellitus. Concerning patients' usual medications, the median number of drugs was seven (interquartile range 4-9.2). Polypharmacy (five or more drugs per day) was present in 348 residents $(72.5 \%)$.

Concerning the management of COVID-19, 375 (78.1\%) received azithromycin, which was combined with hydroxychloroquine in 117 cases $(24.4 \%)$. No resident received hydroxychloroquine without azithromycin. In addition to azithromycin, 346 residents $(72.1 \%)$ also received another antibiotic (ceftriaxone [59.4\%], penicillin [7.9\%]). Seventeen residents $(3.5 \%)$ received only ceftriaxone. Preventive anticoagulation was initiated in 203 residents (42.3\%). In contrast to Cattaneo et al. [1], no resident received immunosuppressants or lopinavir/ritonavir because of the frailty of the population and ambulatory management. Only $23.9 \%$ (one missing data) of residents were hospitalized in a COVID-19 acute unit.

Of the 480 patients, 256 residents (53.3\%) were exposed to at least one potentially severe DDI, similarly to Cattaneo et al. [1]. Table 1 describes the type of potential major DDIs 
Table 1 Type of major DDIs ( $N=256$, no missing data)

\begin{tabular}{lcc}
\hline DDIs & $N(\%)$ & Potential adverse event \\
\hline Azithromycin + antidepressants & $87(34)$ & Increased risk of QT prolongation \\
Azithromycin + antipsychotics & $93(36.3)$ & Increased risk of QT prolongation \\
Azithromycin + statins & $11(4.3)$ & Increased risk of rhabdomyolysis \\
Azithromycin + antiarrhythmics & $18(7)$ & Increased risk of cardiotoxicity \\
Other azithromycin-induced DDIs & $3(1.2)$ & AVK overdose and bleeding \\
Hydroxychloroquine + proton pump inhibitors & $36(14.1)$ & Decreased digestive absorption \\
Hydroxychloroquine + antidepressants & $28(10.9)$ & Increased risk of QT prolongation \\
Hydroxychloroquine + antipsychotics & $47(18.3)$ & Increased risk of QT prolongation \\
Hydroxychloroquine + thyroid hormones & $19(7.4)$ & Hypothyroidism \\
Hydroxychloroquine + antidiabetics & $11(4.3)$ & Hypoglycemia \\
Other hydroxychloroquine-induced DDIs & $20(7.8)$ & Risk of seizures
\end{tabular}

$A V K$ antivitamin $\mathrm{K}$ agonists, $D D I s$ drug-drug interactions

Table 2 Type of contraindications $(N=124$, no missing data)

\begin{tabular}{lr}
\hline Contraindicated DDIs & $N(\%)$ \\
\hline Azithromycin, hydroxychloroquine & $117(94.3)$ \\
Intramuscular ceftriaxone injection and anticoagulant & $34(27.4)$ \\
$\quad$ drugs for curative anticoagulation & \\
Escitalopram, hydroxychloroquine & $10(8.1)$ \\
Hydroxyzine, hydroxychloroquine & $3(2.4)$ \\
\hline
\end{tabular}

$D D I s$ drug-drug interactions

noted by the addition of treatments for COVID-19. The drivers were azithromycin and hydroxychloroquine. These treatments and antidepressants/antipsychotics were the most frequent DDIs. In effect, psychotropic medicines are commonly used in nursing homes, despite the harmful association in elderly people [5]. As reported by Cattaneo et al. [1], the large majority of DDIs increased the risk of QT prolongation. Contraindications were observed in 124 residents (25.8\%) based on the drug information database Drugs.com (Table 2). The main contraindication was the association of hydroxychloroquine and azithromycin (94.3\%) and, like Cattaneo et al. [1], the majority of contraindicated DDIs observed had an increased risk of cardiotoxicity (QT prolongation, torsade de pointes, or ventricular arrhythmias).

We confirmed that in nursing homes a dramatic increase in antibiotic consumption ( $81.6 \%$ of the residents) was seen, explained in particular by azithromycin prescription due to its potential immunomodulatory activity. However, larger studies have shown no benefit, and this treatment was no longer recommended as of June 2020 [6, 7]. Concerning hydroxychloroquine, recent clinical data documented a lack of benefit in patients treated with hydroxychloroquine alone or in combination with azithromycin and warned about potential adverse effects $[8,9]$. In the study by Kelly et al.
[9], the risk of QT prolongation was significantly higher in patients treated with hydroxychloroquine and azithromycin.

In our study, only 45 residents $(38.5 \%)$ treated with hydroxychloroquine and azithromycin had electrocardiogram screening with QTc assessment and QT prolongation present before COVID-19 treatment initiation in $17.1 \%$ of residents. Adverse events were notified in 44 medical records (9.2\%). The most common adverse event was QT prolongation $(29.5 \%)$ but this result is underestimated because of the lack of electrocardiogram monitoring and the retrospective nature of our study.

In conclusion, in nursing homes, a high risk of severe DDIs due to comorbidities and polypharmacy with numerous QT-prolonging medications and the lack of close medical follow-up highlight the need for caution when prescribing therapy for COVID-19. Our results support the need to carefully evaluate the benefit/risk ratio of treatments for COVID-19 in elderly patients. We agree with Cattaneo et al. [1] that respect for the contraindications, appropriate use, and close monitoring must be applied to allow the safe use of these drugs.

Acknowledgements The authors of this article acknowledge the residents, healthcare workers, and administrators in the participating nursing homes. It was a true privilege for the hospital to be partnered with these nursing homes.

\section{Disclosures}

Funding No sources of funding were used to conduct this study or prepare this article.

Conflicts of interest Florian Correard, Anne-Laure Couderc, Robin Arcani, Joris Weiland, Anais Courcier, Charlotte Berard, Charlène Miola, Julie Berbis, Patrick Villani, and Aurélie Daumas have no conflicts of interest that are directly relevant to the content of this article. 
Ethics approval The study was authorized by The National Institute for Health Data (number INDS-MR 3109280520) and conducted in accordance with MR-004 reference methodology approved by the National Commission for Information Technology and Civil Liberties (French CNIL). In accordance with French legislation, formal approval from an ethics committee was not required for this type of retrospective non-interventional study based on the reuse of already recorded data.

Consent to participate Not applicable.

Consent for publication Not applicable.

Availability of data and material The data are available from the corresponding author upon reasonable request.

Code availability Not applicable.

Authors' contributions A-LC, FC, and AD had full access to all of the data in the study and take responsibility for the integrity of the data and the accuracy of the data analysis. Concept and design: A-LC, FC, $\mathrm{PV}$, and $\mathrm{AD}$. Acquisition, analysis, or interpretation of data: all authors. Drafting of the manuscript: A-LC, FC, and AD. Critical revision of the manuscript for important intellectual content: all authors. Statistical analysis: JB. Administrative, technical, or material support: A-LC, FC, AD. Supervision: PV.

\section{References}

1. Cattaneo D, Pasina L, Maggioni AP, Giacomelli A, Oreni L, Covizzi A, et al. Drug-drug interactions and prescription appropriateness in patients with COVID-19: a retrospective analysis from a reference hospital in northern Italy. Drugs Aging. 2020;37:925-33.

2. Hodge C, Marra F, Marzolini C, Boyle A, Gibbons S, Siccardi $\mathrm{M}$, et al. Drug interactions: a review of the unseen danger of experimental COVID-19 therapies. J Antimicrob Chemother. 2020;75:3417-24.

3. Onder G, Liborio Vetrano D, Cherubini A, Fini M, Mannuccio Mannucci P, Marengoni A, et al. Prescription drug use among older adults in Italy: a country-wide perspective. J Am Med Dir Assoc. 2014;15(531):e11-15.

4. Maher R, Hanlon J, Hajjar E. Clinical consequences of polypharmacy in elderly. Expert Opin Drug Saf. 2014;13:57-65.

5. Sawan M, Jeon YH, Chen TF. Relationship between organizational culture and the use of psychotropic medicines in nursing homes: a systematic integrative review. Drugs Aging. 2018;35:189-211.

6. Rosenberg ES, Dufort EM, Udo T, Wilberschied A, Kumar J, Tesoriero J, et al. Association of treatment with hydroxychloroquine or azithromycin with in-hospital mortality in patients with COVID-19 in New York State. JAMA. 2020;323:2493-502.

7. Furtado RHM, Berwanger O, Fonseca HA, Corrêa TD, Ferraz LR, Lapa MG, COALITION COVID-19 Brazil II Investigators, et al. Azithromycin in addition to standard of care versus standard of care alone in the treatment of patients admitted to the hospital with severe COVID-19 in Brazil (COALITION II): a randomised clinical trial. Lancet. 2020;396:959-67.

8. Alvi MM, Sivasankaran S, Singh M. Pharmacological and nonpharmacological efforts at prevention, mitigation, and treatment for COVID-19. J Drug Target. 2020;28:742-54.

9. Kelly M, O'Connor R, Townsend L, Coghlan M, Relihan E, Moriarty $\mathrm{M}$, et al. Clinical outcomes and adverse events in patients hospitalized with COVID-19, treated with off-label hydroxycholoroquine and azithromycin. Br J Clin Pharmacol. 2021;87(3):1150 4. https://doi.org/10.1111/bcp.14482. 